\title{
An empirical method for the study of exemplar explanations
}

\section{Mads Goddiksen}

Centre for Science Studies, Department of Physics and Astronomy, Aarhus University, Denmark

E-mail: mads.goddiksen@ivs.au.dk

\begin{abstract}
The most common way of studying explanations in philosophy of science and science education is through case studies. Recently these have been supplemented with studies based on empirical methods. This chapter provides an empirical method for collecting and comparing exemplar explanations across scientific disciplines with the aim of exposing possible qualitative differences between them. The method is based on the use of science textbooks as sources of explanations. I discuss a number of possible strategies for identifying explanations in these sources, and specify a set of reliable linguistic indicators that can be used for this purpose. A pilot study is presented to illustrate the method and its limitations.
\end{abstract}

\section{Introduction}

Within philosophy of science there has been considerable interest in scientific explanations for several decades. There is general agreement that constructing and evaluating explanations is a very important part of what practicing scientist do, but there is less agreement on how and why they do it. Many of the classical studies of scientific explanations aim to answer the question of how scientific explanations differ from nonscientific explanations by providing accounts of the characteristics of scientific explanations regardless of which part of science they originate from (Friedman, 1974; Hempel, 1965; Salmon, 1998). Against this overall project Van Fraassen argued that there are no interesting common features in explanations across all the sciences (Van Fraassen, 1980). Others have argued that although explanations are important in all the sciences, the standards for what counts as a good explanation can change as disciplines change over time (McMullin, 1993) and it is widely recognized that disciplines coexisting at one period of time have different standards for what counts as a good explanation (Godfrey-Smith, 2003, ch. 13; Woodward, 2011; Woody, 2003). This opens for more specific studies of explanations from specific disciplines (e.g. mechanistic explanations in the life science (Machamer, Darden \& Craver 2000)) and comparative studies across disciplines.

Given that explanations play a key role in scientific practice, learning to construct and evaluate explanations should also be an important part of any science education. This has also been recognized within the science education literature (Braaten \& Windschitl, 2011) and researchers in this field have therefore taken an interest not only in scientific explanations given by scientists to their peers and how these differ from every day explanations but especially in explanations intended for students. One conclusion from this research is that there is a need for more explicit teaching on how to construct high quality explanations (see e.g. (Solomon, 1995; Peker \& Wallace, 2011)). 
Many studies in the science education literature refer to studies from other disciplines such as philosophy, linguistics or discourse analysis (Edgington \& Barufaldi, 1995; Rowan, 1988; Unsworth, 2001) as a general framework for the development of a more detailed analysis of explanations from textbooks or teaching situations. It is interesting to note that the discussions about the potential paradigm dependence of the criteria for high quality explanations have been somewhat overlooked in the science education literature. This means there is a risk that explicit teaching in how to construct good explanations will not be sufficiently nuanced.

In this chapter I outline a methodology that can be used to study and compare explanations from different disciplines drawing on both science education and philosophy of science. More specifically, the main aim of this chapter is to formulate an empirical method based on gathering and analyzing the exemplar explanations practicing scientists use in education. Following Kuhn (1996), exemplar explanations are explanations presented to coming members of a scientific community both in order to help them understand a given explanandum, but also to display what a good explanation looks like (see also (Treagust \& Harrison 1999)). These explanations thus play an important in teaching coming members of a scientific community how to construct good explanations. By comparing the exemplar explanations it is possible to identify and describe possible differences in standards for good explanations across disciplines. The method presented in this chapter is therefore aims developed to provide a descriptive account of exemplar explanations that is sensitive both to differences in practices between different disciplines and to differences in practices between different educational levels ${ }^{1}$.

To argue for the value of empirical studies of explanations, and to see how my method differs from previous empirical approaches to the study of explanations, I start out by discussions Andrea Woody's empirically based account of explanations in chemistry (section 2) and the methodology presented by Zoubeida Dagher for the study of explanations given by science teachers (section 3 ).

\section{Andrea Woody's account of explanations in chemistry}

Empirical studies of explanations are rare in the philosophy of science literature. An important exception is the empirically based account of explanation in chemistry that has been developed by Andrea Woody (2004a; 2004b). Although the aim of Woody's study of explanations is quite different from mine, it is interesting to discuss her general argument for choosing an empirical method.

\footnotetext{
${ }^{1}$ As argued elsewhere (Goddiksen 2013), the results of such a comparison will, for instance, be valuable to educators aiming to teach interdisciplinary problem solving. One of the epistemological challenges faced in interdisciplinary problem solving is to navigate the differences in standards for good explanations across different disciplines and knowing what these differences are will ceteris paribus make this process easier.
} 


\subsection{Why choose an empirical method}

Woody has chosen an empirical method for two reasons ${ }^{2}$. One is her wish to give a highly descriptive account of explanations in chemistry (2004a, pp. 17-18). The other reason is that she sees a flaw in the argumentation in earlier case based studies that she wants to avoid.

According to Woody the typical way to analyze explanations in philosophy of science has been through inductive arguments starting from a small set of paradigmatically successful explanations (2004a, p. 36). The structure of these inductive arguments can roughly be represented by what we might call the classical pattern of argument or just CP (ibid.):

\section{1. $a$ is a successful explanation of $b$}

1.2. The basic, or most noteworthy, characteristics of $a$ are $\{j, k, l, \ldots\}$.

1.3. Members of the set $\{j, k, I, \ldots\}$ are (quasi)tokens of corresponding types $\{J, K, L, \ldots\}$

Infer by generalization

$\mathrm{C}_{\mathrm{CP}}$ : The requirements for successful explanation are $\{\mathrm{J}, \mathrm{K}, \mathrm{L}, \ldots\}$

We can now go on to test $C_{c p}$ by analyzing other paradigmatically successful explanations.

Friedman (1974) seems to be using a version of this pattern when he argues that the kinetic theory of gasses, which is a "typical scientific theory" (p. 14), effects a significant unification of our view of nature. He then concludes that "this is the crucial property of scientific theories we are looking for; this is the essence of scientific explanation" (p. 15). Friedman deviates from the CP by omitting the last part, where you test your conclusion on a different case.

The easiest way to argue against conclusions drawn from CP is to find a paradigmatically successful explanation $c$ whose characteristics are not (quasi)tokens of $\{J, K, L, \ldots\}$. An example of this strategy can be found in (Weatherall, 2011) where an explanation of why inertial and gravitational mass are equal in Newtonian mechanics is discussed. Weatherall argues that the essential features of this explanation (and others like it) are not captured by any causal theory of explanations, neither by Kitcher's unificationist account nor by Hempel's Deductive-Nomological account of explanation. Thus it represents a counter example to the inductive foundations of these theories.

Woody's critique of this way of analyzing scientific explanations is that the justification for the choice of paradigmatically successful explanations is deficient. This would not be a problem if it was possible to identify a relatively large set of candidates that everyone (or at least all philosophers of science) would intuitively accept as successful explanations. But unfortunately we do not have such a set. This has led to what Woody sees as a rather pointless debate:

\footnotetext{
${ }^{2}$ Woody actually calls her method "quasi-empirical" (2004a, p. 13). This seems reasonable given that her empirical material is limited to just one textbook. However there is nothing semi-empirical about the methodology employed in the study.
} 
[Philosophers] quarrel famously over a set of reputed, but still disputed, "counterexamples": the flagpole and the shadow, the ink spill on the carpet, leukemia and radiation exposure, John Jones' recovery from pneumonia. This dispute cannot possibly be settled in this manner. (Woody, 2004a, p. 15)

It seems then that claiming that a certain case is a good example of a successful explanation is far from trivial. This means that the choice of cases needs additional justification. According to Woody (2004a, p. 39), the only theoretical justification that can be offered for the choice of the examples is an appeal to pre-analytic intuitions about the general nature of (successful) explanations. However, Woody argues that this kind of justification would make the account viciously circular:

It is precisely the general nature of explanation [...] we are attempting to determine via this argument. Thus either we are involved in a vicious form of circular reasoning or we need some independent means of justifying [this premise]. (Woody, 2004a, p. 39)

Hence Woody's second argument for choosing an empirical method is to avoid this kind of vicious circular reasoning (hereafter referred to as Woody's circularity objection). Here an empirical method means identifying sources of explanations that practicing scientists deem successful and extract the explanations from these. This way, according to Woody, it is possible to avoid the kind of vicious circular reasoning involved in the earlier studies.

The question is of course a) what sources to choose, and b) how to identify the explanations in the sources? Woody's answer to a) is that science textbooks are highly useful sources if used correctly (see (Woody, 2004a, pp. 18-19) for details). I agree with Woody that science textbooks are valuable sources when studying scientific explanations empirically. More specifically I argue in section 2.3 that they valuable given the purposes of this chapter.

With respect to b), Woody does not give an explicit answer to how explanations in textbooks can be identified. I discuss approaches taken by others in section 3-5 and present my own answer in section 6.

Assuming (for now) that there is a good answer to these two questions at hand, can an empirical method like the one developed below avoid Woody's circularity objection? To the extent that Woody's circularity objection is valid I believe the method outlined below will address the objection.

The aim of the methodology developed in this chapter is to identify explanations from a given discipline with a certain quality, namely explanations that are accepted by practicing scientists from this discipline as good explanations to give to students. If the identification of these explanations is based on pre-analytic intuitions about the nature of explanations with this quality the study would certainly be viciously circular. But such assumptions need not be made. As outlined below I suggest is that the focus should rather be on identifying explanations in sources that we have independent reason to believe contain only explanations with the desired quality. Thus I avoid the vicious normative circle where a normative claim about the nature of good 
explanations to give to students is based on intuitions about what constitutes a good explanation to give to students. However the argument is still based on a descriptive circle. I will still have to assume something about the nature of explanations in order to identify them in the sources. Thus the description of the explanations in the sources must still rely on an explicit or intuition based pre-analytic description of explanations that enables us to recognize explanations in the sources, so clearly the method developed here involves circular reasoning. But this kind of descriptive circularity, which is present in any empirical investigation, is not vicious (Nersessian, 1995)

So if Woody's objection is interpreted as a reference to only the vicious normative circularity involved in earlier studies, the method developed here will not be targeted by the objection. This of course will only be the case if satisfactory answers to questions a) and b) can be provided along with independent reasons for why the chosen sources contain only the desired kind of explanations.

\subsection{Identifying good sources}

What would be a good source of exemplar explanations given to students by practicing scientists from a given discipline? Since my primary interest here is in explanations that are widely accepted within the discipline as being of high quality it seems reasonable to look mainly to written sources that have been through some kind of critical review. These kinds of written sources fall into two general categories: 1) peer reviewed journal articles and other documents that aim to convey novel results to practicing scientists, and 2) textbooks and other documents that aim to convey established knowledge from the discipline to students (among others).

Although an explanation presented in a journal article has been through peer review, it is not necessarily uncontroversial. Some articles do reach such a high status within a discipline that they become widely used as prototypes of what a good scientific article and a good scientific explanation is. If these can be identified they might prove valuable, but the primary source of exemplar explanations are the widely used textbooks from the disciplines under investigation ${ }^{3}$. These sources are written explicitly with teaching in mind and all the explanations in them have been carefully selected as suitable explanations to give to students at a given level of education. These explanations are thus constrained both by the educational level of the intended audience and the standards for good explanations in the given discipline (Treagust \& Harrison 1999). Arguably, the constraints from the level of the intended audience are most prominent in lower level textbooks, whereas most advanced textbooks are primarily constrained by the standards for good explanations in the relevant discipline. In order to gain knowledge about how practicing scientists' explanations to students develop as the students progress through their education, it will therefore be important to make sure that the selection of textbooks includes both introductory and advanced texts.

\footnotetext{
${ }^{3}$ Textbooks are not only particularly suited for studies of explanations to students, they are also more generally good sources of explanations. Indeed, anyone interested in widely accepted explanations should be interested in textbooks, since the explanations found in scientific articles are not necessarily uncontroversial. Furthermore textbooks are useful for a study (like Woody's) that aims to answer why explanations are so important in scientific practice, because textbook explanations can provide clues as to why and how explanations are valuable to practitioners since one of the aims of a textbook is to show future practitioners how to use the tools of the discipline. (Woody, 2004a p. 18)
} 


\subsection{How to identify explanations in the sources selected?}

Once a set of sources is identified, the next step is to identify the explanations in them. This is complicated by the fact that although a textbook may contain many explanations of natural phenomena, experimental procedures etc., they do not necessarily contain just explanations. For one a textbook may have to devote space to describe various explananda, which may include the description of experimental setups and puzzling data. Furthermore, many explanations are cashed out in terms of entities and activities, some of which may not be familiar to the student (Machamer, Darden \& Craver 2000). Significant portions of a textbook may thus have to be devoted to introducing entities (abstract and concrete) or activities that feature in explanations, but are not necessarily explained themselves to a significant extent. For instance: In order to be able to explain the workings of a Scanning Tunneling Microscope it will be important to introduce the activity "quantum tunneling" as well as entities such as electrons that perform these activities. Ogborn and collaborators thus found that in the classroom, science teachers in secondary school spend much time on "the construction of entities" (Ogborn et. al. 1996, ch. 3) used in explanations. Looking closer at their examples, reveals that this often involves introducing the activities they are involved in as well (p. 39). In addition to entities and activities that are parts of mechanisms, textbooks may also introduce a number of models that can be used as parts of explanations of other things. Some of these introductions will be explanations, but it is not possible to explain everything. Some entities, activities and models are likely to be introduced as black boxes that may or may not be explained elsewhere. When studying textbooks the investigator therefore cannot take for granted that every passage of a textbook is meant to explain and provide understanding. Passages that are not meant to be explanatory need not live up to the standards of good explanations in place within the discipline, and including them in the sample may thus give a distorted picture of what is considered a good explanation to give to students. It is therefore necessary to have some form of criteria that can be used to identify the explanations that are contained in the sources. There seem to be a number of ways in which an investigator may approach this challenge. One is to rely on pre-analytic intuitions about good explanations to give to students. Relying on these would of course make the empirical argument just as viciously circular as the case-based studies that Woody criticizes. There is thus a general worry that simply making an empirically based claim is not in itself sufficient to overcome Woody's circularity objection. If the claim is based on a biased dataset gathered using a spurious method it should not be considered any better than an invalid theoretical argument. In order to overcome Woody's circularity objection it is therefore important that a way of identifying explanations in textbooks is found, that is not dependent on the investigator's intuitions about what a good explanation is.

Woody's writing does not help us much in this direction. Although Woody (2004a) presents some examples of explanatory structures - theories, parts of theories, pictures, diagrams and other structures that play important roles in explanations - which she identified in a general chemistry textbook (Mahan \& Myers, 1987), she does not tell us how she identified these. This is not necessarily a problem for Woody, as one need not be able to identify explanations in textbooks in order to identify explanatory structures in them. (Although one of course needs to be able to argue that these structures are actually used in explanations somewhere.) But it does mean that it is necessary to expand on Woody's account in order to reach an answer to the circularity problem pointed the Woody points to. 
Looking to the few other empirical studies of explanations suggests two possible strategies for identifying explanations in textbooks. One is to rely on pre-analytic intuitions on what an explanation is - regardless of whether it is good or bad, another is to use explicit criteria for - perhaps even a definition of - when a passage in a textbook can be considered an explanation. I discuss the former of these options in the next two sections and the latter in section 6.

\section{Studying science teachers' explanations}

Zoubeida Dagher and George Cossman have categorized explanations given by science teachers in junior high schools, based on extensive empirical material (Dagher \& Cossman, 1992). In an earlier article (Dagher, 1991) Dagher provides insight into how these explanations were identified and classified. Dagher notes that identifying explanations in sources (in her case recordings of classroom discourse) can be difficult:

While the purpose of the analysis was perfectly clear, the question about what constituted an explanation, particularly a teacher explanation, became more obscure. [...] The literature that was reviewed presented serious dilemmas. In the case of educational theory, the adoption of any particular definition appeared to fail to discriminate between 'explanations' and other categories of verbal behavior. In the case of philosophy of science, definitions tended to restrict the sense of explanation so as to eliminate instances that seemed to be legitimate teacher explanations. (Dagher, 1991, p. 68-69)

So instead of combing the transcripts with a definition Dagher chose to search the transcripts for passages that intuitively "looked like" explanations believing that it was possible to justify the selection later on (p. 69). When personal intuitions were unclear Dagher resorted to the "conscious and tacit entertainment of various literature based 'attributes' of explanations" (p. 70).

For a researcher who is philosophically minded and who knows the field under study very well this approach is likely to be productive. However, for the purposes of this study two concerns can be raised.

First of all this approach explicitly identifies what the investigator deems explanatory, and unless the investigator is highly familiar with the discipline under study this may differ from what practicing scientists in that discipline deem explanatory. This could be tested by asking practicing scientists if they agree with what the investigator has identified. But if this step is needed in order to get a useful result, why not go all the way and simply leave it to the scientists to identify the explanations in the sources? (see section 5).

Secondly, Dagher admits that the results of her investigation would probably look different if the analysis was performed by someone else (Dagher, 1991, p. 76). This is of course often the case with such interpretive studies, and it is not necessarily a problem, especially if it is mainly the finer details in the conclusions that depend on who performed the interpretive study. However, as we saw in section 2.1 part of the reason why, at least philosophers, quarrel so much about explanations is that the differences in intuitions about explanations 
among philosophers are rather substantial. This indicates that it may not just be the finer details, but the entire outcome of the study that becomes dependent on who performs the study if it relies heavily on the intuitions of the investigator. This is certainly something one should aim to avoid to the extent possible. At least it should be made as transparent as possible how the intuitions of the investigator affected the outcome of the study.

For these reasons it will be preferable to base the study on explicit criteria that can be judged by others, or alternatively to base it on the intuitions of the practicing scientists themselves.

\section{Conclusions on Woody and Dagher}

The above discussion shows that one way to expose possible qualitative differences in exemplar explanations from different disciplines is to use a varied selection of textbooks as sources of explanations. When explanations in these sources have been identified the explanations from the different disciplines can be characterized and compared. While these later stages present their own challenges, my main focus here is how to identify explanations in the selected textbooks.

I have argued that it is important to explicate how this is done in order to construct a strong empirical argument. Furthermore I have argued that basing the identification largely on the investigator's intuitions will lead to results that are investigator dependent to an extent that is undesirable.

I will therefore proceed to discuss two different (but not mutually exclusive) ways to identify explanations in textbooks:

1. Ask practicing scientists from the discipline under investigation to go through the texts and identify explanations.

2. Make explicit assumptions about reliable indicators of explanations in textbooks, use these to identify the explanations in the textbooks.

I will argue (in the following section) that the first option could provide some very interesting insights if combined with follow up interviews, but that it is more suited for providing a detailed account of explanations within one specific discipline than for mapping differences in explanations from different disciplines.

In section 6 I will discuss the theoretical advantages and limitations of the second option and provide some insight into the practical challenges as I discuss the results of a pilot study based on textbooks from chemistry and physics.

\section{Ask practitioners to identify explanations}

One way to investigate what practicing scientists deem to be explanations in a selection of textbook material could be to ask practicing scientists themselves to identify explanations in the material. This approach certainly has advantages. First of all, it ensures that the explanations found are indeed deemed to be explanations by practicing scientists, not just by philosophers or other outsiders. As I have argued, this satisfaction is not trivial 
to obtain through other means. Secondly, the investigator can avoid making assumptions about the nature of explanations, which might be desirable for investigators that are worried about Woody's circularity objection.

The downside to this approach is that it is likely to be very resource consuming and thus difficult to carry out in practice.

Furthermore, since disagreements between the scientists about which passages in the texts are explanations are to be expected, it will be necessary to develop a way to decide when enough participants have marked a passage as an explanation to qualify as widely accepted as an explanation. If the requirement is that everyone approached has to have marked a specific passage as an explanation before it can be allowed into the pool of data then there is likely to be little data unless the number of sources that the science practitioners have to study is very high. Relying on a simple majority will on the other hand be too permissible since the minority might contain a significant number of the most experienced teachers or specialized researchers from the area of the discipline from which the candidate explanation originates.

One way to overcome these difficulties would be to gain more knowledge about the researchers (and about their position in their field) and also about why they chose the different passages for example by interviewing the practitioners afterwards or inviting them to "think aloud" while identifying explanations in textbook samples. Adding this extra layer to the investigation could yield a more detailed picture of explanations in the disciplines under investigation but is also likely to be highly time- and resource consuming. Furthermore, the more detailed picture of explanations in the disciplines to be compared that this approach may yield is not strictly necessary for my current purposes. If there are significant differences across scientific disciplines then these differences are the ones that will be most relevant both in a philosophical and educational perspective, and these should be detectable through a comparison of a less detailed picture of explanations from the disciplines compared.

I will therefore go on to discuss the possibility of identifying explanations using a set of reliable indicators in order to assess whether the theoretical and practical limitations of this approach are more suited to the purposes of the current study.

\section{Using reliable indicators}

As argued in section 2.1, a descriptive study of exemplar explanations does not necessarily result in a vicious circle if it relies on reliable indicators or even a definition of what constitutes an explanation to identify explanations in the textbooks. What I have not yet considered is which definition or indicators to rely on and whether it is practically convenient to proceed in this way.

Considering the question about indicators first, we note that explanations may be defined with reference to their function, and with reference to their structure. I have already made assumptions about an essential function of explanations, namely that successful explanations provide understanding. So one possibility is to 
start from this assumption and then investigate how understanding is gained in the discipline in question. ${ }^{4}$ The problem with this assumption is (as Salmon also noted (1998, p. 126)) that they do not help further investigations if they are kept too general. The nature of understanding and intelligibility is not better mapped than the nature of explanation, so simply assuming that one provides the other does not help. A more detailed description of what is meant by understanding and how an explanation can provide this understanding is required for this approach to prove useful. If this is done on the basis of theoretical arguments, one could end up identifying what ought to provide understanding from a theoretical perspective rather than identifying what practicing scientists deem to be good explanations to give to students ${ }^{5}$. This is exactly what I aim to avoid.

Alternatively, one might assume something about the linguistic indicators of explanations ${ }^{6}$. For instance, it may be possible to search for specific language structures, or even certain key words.

Against this strategy, Stephen Draper has argued that there are no linguistic traits common to all explanations (Draper, 1988). Some, but not all, will be answers to explicitly posed why- or how-questions. Many will contain the word 'because', but some will not. More generally, Draper argues that there are no words or sentence structures that can be called necessary for explanations. Thus "[...] a search of a transcript for their occurrence will not pick out anything like the complete set of the explanations present" (Draper, 1988, p. 20). Draper does admit that the presence of a word like 'because' can be seen as a sufficient condition for the presence of an explanation (p. 19). So there is nothing in the theoretical arguments that prevents us from saying that a search for keywords in a textbook could yield a good sample of the explanations found in the text. And this is really all we need! The question now is whether the sample will be big enough to be practically useful, and whether we have reason to think that the sample will not reflect the diversity in the explanations in the textbooks because the keyword search leaves out certain important types of explanations?

I will discuss the former question of sample size in detail when I present my pilot study in section 6.3. The answer to the latter question depends on which keywords are used. Before answering this question I will therefore have to elaborate a bit more on which keywords should be used.

\footnotetext{
${ }^{4}$ See (Chambliss, 2001) for an example of a study of explanations based on assumptions about understanding.

${ }^{5}$ A different kind of objection to this approach might also be raised: Even if it can be safely assumed that any good explanation will increase the reader's understanding of the explanandum, this does not mean that a good explanation is necessary for an increase in understanding. Thus we will be making the fallacy of affirming the consequent if we claim to have found explanations by identifying passages that increases the readers understanding. Lipton (2009) has explored other sources of understanding (for instance thought experiments), and this potential objection could be overcome by simply assuming that Lipton's list of sources of understanding is exhaustive. If a textbook passage increases the readers understanding and does not belong to one of the other sources of understanding on Lipton's list, it can safely be assumed that an explanation has been found.

${ }^{6}$ Rowan (1988) has also discussed the advantages and challenges related to the study of explanations through assumptions about either their function or their structure. She argues that if the purpose of the study is to improve teaching, then assumptions about the function of explanations is preferable, but unfortunately she does not give us any hints as to how the practical problems associated with this approach might be overcome.
} 


\subsection{Introducing the keywords}

Overton (2013) used text mining methods based on keyword searches to assess the importance and abundance of explanations in the journal Science. Overton relied exclusively version of the words 'explain', 'explanation' and 'explicate' as keywords indicating the presence of explanations in the articles. Although 'explanation' and 'to explain' are perhaps the most obvious candidates for a list of reliable indicators for the presence of an explanation, it seems unnecessarily restrictive to rely solely on these, if the aim is to identify a large and diverse set of explanations.

There is a broad consensus both in philosophy and in science education that the primary function of explanations (especially those in textbooks) is to provide understanding (de Regt, Leonelli, \& Eigner, 2009; Rowan, 1988). So although one cannot search for passages that will provide understanding to the reader, one can search for those passages that the author(s) of a textbook has explicitly stated should provide understanding ${ }^{7}$, passages like "To understand this ..." or "this helps us understand ...". Thus, versions of the words 'to understand' and 'understanding' should be added to the list of keywords to be searched.

As mentioned earlier, Draper (1988) (among others) acknowledges that 'because' is a reliable indicator for the presence of an explanation, and therefore can also be added to the list of keywords.

Finally, one can search for answers to explicit explanation seeking questions, at least if it is possible to specify more concretely the nature of such a question. When philosophers discuss explanations they often focus on answers to why-questions (Goodwin, 2003; Salmon, 1998; Van Fraassen, 1980), but it is also widely recognized that being an answer to a why-question is not a necessary condition for being an explanation. In addition, certain how-questions are often highlighted as explanation seeking. Mechanistic explanations, for instance, have been described as answers to how-questions (Machamer, Darden \& Craver 2000). Furthermore, certain what-questions may also be explanation seeking. The geologist might, for instance, try to explain what went on in the Cambrian explosion. In general there is no reason to believe that answers to questions involving certain interrogatives can be excluded as being explanations. (Draper, 1988; Faye, 1999).

The reason why answers to questions other than why-questions are not discussed as much by philosophers as answers to why-questions may be that it becomes less clear when answers to these kinds of questions are explanations. Whereas we can treat all relevant answers to why questions as explanations we cannot automatically do the same with answers to questions involving other interrogatives. For how-questions it is still relatively uncontroversial that questions about how things work are explanation seeking whereas it is more unclear whether how-much-questions like "how much ascorbic acid does a normal person need per day to avoid scurvy?" are explanation seeking. For other interrogatives it becomes even more difficult to say whether a question is explanation seeking or not simply based on the wording of the question.

Thus a reasonable way to proceed would be to start out by searching for answers to why- and how-questions (excluding how-much-questions), the word "because", and all versions of the words 'explanation', 'to explain', 'to understand' and 'understanding', and use the data gained in this search to sketch the characteristics of

\footnotetext{
${ }^{7}$ Bearing in mind the possible objection raised in note 5 about the possibility of other sources of understanding.
} 
exemplar explanations in a given discipline. This could then be used to analyze answers to other kinds of questions yielding an even more detailed picture, and so on until a sufficiently detailed account that enables one to make comparisons to other disciplines is at hand.

\subsection{Concerns about diversity}

Having identified some useful keywords for seeking explanations in texts, I will return to the question of whether one might miss important types of explanations by relying on these keywords. Since the list of keywords contains no necessary conditions for the presence of an explanation, it is difficult to argue decisively that every type of explanation will be found. However one can argue that the keyword search will detect at least as many types of explanations as other methods. Take for instance the ten categories that Dagher and Cossman present (1992, pp. 364-366), one can argue that a keyword search would identify all of these categories.

After describing the characteristics of each of the categories Dagher and Cossman present an example of each of the ten types of explanations taken from their transcripts. Half of these examples contain either a whyquestion or the word 'because'. Two further categories (tautological and practical explanations) are partly defined as answers to how/why questions. So in these cases a search for key words presented above in the transcripts would not only identify explanations of the same type, but it would even identify the examples presented by Dagher and Cossman.

Is there reason to believe, that explanations of the remaining three types could not be found through a keyword search? Two of the remaining categories will be familiar to most readers: teleological explanations and explanations that explain through analogy. Such explanations can be and are given as answers to why-and how-questions.

Last but certainly not least an important type of explanation in textbooks appears as descriptions of what happens, rather than how things work, or why they happen (as Woody has also pointed out (Woody, 2003, p. 23)). Dagher calls these genetic explanations, and they present quite a challenge for anyone interested in explanations who want to distinguish between explanatory and non-explanatory descriptions. Explanatory descriptions that are also explanations are considered to provide (genuine) understanding, and therefore should be identifiable through a search for the term 'understanding' or 'to understand', given that these terms are in fact used regularly in the textbooks. As we shall see in the following section this is in fact the case, at least in introductory textbooks.

All in all, this shows that the sample of explanations found through a keyword search as outlined above will be at least as diverse as a sample gained through an intuition based search.

Let me finally illustrate how the approach outlined so far could be used in practice.

\subsection{Some results from a pilot study}

To test the practical limitations of the first steps in the key word based approach outlined above I performed a small pilot study. I chose to focus on thermodynamics. This topic is central to both physics and chemistry, and 
there is an abundance of textbooks on the market aimed at audiences ranging from novices to experts. I chose a textbook from each end of this spectrum to see whether the usefulness of my approach depended on the intended audience of the textbooks. More precisely the sample studied consisted of chapters 17-20 (both included, 126 pages in total) from University Physics (Young \& Freedman, 2010) which is a very widely used introductory textbook in physics and chapters 2-8 (107 pages) from an older textbook called Chemical Thermodynamics (Kirkwood \& Oppenheim, 1961) which is "intended to serve as the basis of a senior or graduate course" for chemists ${ }^{8}$.

\subsubsection{Results from the keyword search}

I searched for each of the keywords ('because, answers to why- and how-questions plus versions of 'explanation', 'to explain', 'to understand' and 'understanding') in turn and will comment briefly on the results in the following sections.

\subsubsection{Versions of 'explanation' and 'to explain'}

I first searched the sample for versions of the keywords 'explanation' and 'to explain'. The final count of instances of either of these words in the body text of both samples was only five. Of the five instances two appeared in Chemical Thermodynamics, one in a general introduction to a chapter pointing to specific discussions later on and the other in this later discussion (Kirkwood \& Oppenheim, 1961, sec. 5.2). In University Physics the search yielded three instances in total. Like in Chemical Thermodynamics one instance was in the introduction to a chapter pointing to a discussion later on (Young \& Freedman, 2010, ch. 20).

Another instance was partly stated in a caption to a picture ${ }^{9}$. We are told that "[e]vaporative cooling explains why you feel cold when you first step out of a swimming pool" (Young \& Freedman, 2010, p. 568) and then pointed to a picture of three children in a swimming pool. The caption elaborates a bit on the claim made in the main text:

[...] it may be a hot day, but these children will be cold when they step out of the swimming pool. That's because as water evaporates from their skin, it removes the heat of vaporization from their bodies (Young \& Freedman, 2010, p. 568)

A striking feature of all the discussions linked to this word search is that they are not based on mathematical calculations, but rather on qualitative arguments. To the extent that they do appeal to any general laws these are postulated rather than derived. This is particularly striking for Chemical Thermodynamics, since it aims to "present a rigorous and logical discussion of the fundamentals of thermodynamics [...]" (p. v).

\subsubsection{How-questions}

Learning how is apparently important in introductory physics. Each of the chapters from University Physics states the learning goals of the individual chapter. The three chapters in the sample state a total of 27 different

\footnotetext{
${ }^{8}$ The book was recommended to me by a lecturer in physical chemistry as the most rigorous presentation of chemical thermodynamics that he knew of.

${ }^{9}$ The final instance also appears in the caption to a picture (Young \& Freedman, 2010, p. 564)
} 
things that the student should understand after reading the chapters. 21 of these contain the word 'how', none of them the word 'why'.

Four main categories of how-questions were identified covering most, but not all instances: 1) how-muchquestions, asking for the value of a particular variable under specific conditions, 2) questions about how some natural or artificial thing works - e.g. how a different kinds of thermometers work - , 3) how concepts or laws are related - e.g. the relation between Newton's laws and the ideal gas law - and finally 4) how to answer a how-much-question given specific conditions.

As discussed in section 6.1, answers to questions of type 1) cannot immediately be taken to be explanations, whereas I find it safe to assume that answers to the remaining types of how-questions are explanations when found in textbooks. How-much-questions are by far the most abundant in University Physics, as the exercises following each chapter have a very high proportion of how-much-questions, and most occurrences of 'how' are found here. In the main text around half of the how-questions are how-much-questions. Based on a further analysis of the answers provided to why- and other types of how-questions it is quite possible that some of the answers these how-much-questions may be identified as explanations as well. Focusing for now on the more unambiguously explanation seeking how-questions - there are roughly 5-10 per chapter - one finds that the answers are often quite detailed and relatively technical, taking up more space than for instance answers to why-questions.

Since the vast majority of how-questions in University Physics occur in the exercises, and since there are no exercises in Chemical Thermodynamics, one would expect there to be much fewer how-questions in this more advanced textbook ${ }^{10}$. Indeed the search through Chemical Thermodynamics yielded only three instances. All three instances were of type 3 ) described above about how concepts can be related. Two of the instances figure prominently as the framing questions for an entire chapter and thus indicates that understanding how is important in advanced chemistry as well.

\subsubsection{Why-questions}

The word 'why' appears 992 times in the whole of University Physics ${ }^{11}$, so even though Woody might be right that there is lots of explanatory content that is not phrased as answers to specific questions (Woody, 2003, p. 23) it might turn out that there is simply so much explanatory content in these books that the small fraction of it that is phrased as direct answers to explanation seeking questions will be more than enough for the purposes of this method. In the sample chapters from University Physics the word 'why' appears between five and seven times per chapter. Although this means that 'why' is roughly as abundant as the word 'how' when used in an

\footnotetext{
${ }^{10}$ Furthermore, the physical format of the two books is quite different, so the number of words on a page with no equations or figures is about 50\% higher in University Physics than in Chemical Thermodynamics. Thus even if the key words were equally frequent in the two texts I would still have identified a more instances in University Physics than in Chemical Thermodynamics. One should thus be careful not to read too much into the absolute differences in the number of instances of any of the individual keywords between the two texts.

${ }^{11}$ This makes it more abundant than the words 'explain' and 'understand' which appear 821 and 500 times respectively, but less abundant that the word 'because' which appears 1009 times. The word 'how' occurs 2140 times but as mentioned the majority of these appear in how-much-questions.
} 
explanation seeking context, the answers to why-questions are not as detailed and do not take up as much space. The same is true for Chemical Thermodynamics where the word 'why' appears only once in the sample in order to point to a phenomenon that thermodynamics cannot explain.

It is interesting that explanation seeking how-questions are so much more prominent in both textbooks compared to why-questions since it challenges the approximation commonly used in philosophy that all explanations are answers to why-questions. If further studies can establish that why-questions do not play a significant role in advanced textbooks then perhaps the appropriateness of approximating explanations with answers to why-questions should be re-evaluated.

\subsubsection{Because}

The word "because" is abundant in both texts. A prominent feature of the explanations involving "because" in both samples is their qualitative nature and brevity. Often the explanandum and the explanans are contained within a single or just a few sentences. Take for example the following passage:

The thermal conductivity of "dead" (that is non-moving) air is very small. A wool sweater keeps you warm because it traps air between the fibers. In fact, many insulating materials, such as Styrofoam and fiberglass are mostly dead air. (Young \& Freedman, 2010, p. 571)

In this respect they resemble the explanations found in the search for "explanation" and "to explain".

\subsubsection{Understanding}

The search for the versions of 'understanding' and 'to understand' in Chemical Thermodynamics yielded only two occurrences. One coincided with an instance of because and one coincided with the single occurrence of

"why" pointing to the lack of understanding of the expressions for the entropy of a gas (real or ideal) until the advent of quantum mechanics.

University Physics yielded 55 hits. Versions of the words regularly show up in the introduction to chapters or sections to debut the theory that will be explained later or to stress the importance of certain explanations. For instance the following statement occurs after a passage describing the temperature dependence of the internal energy, $U$, of an ideal gas ( $T$ denotes the temperature):

Make sure you understand that $U$ depends only on $T$ for an ideal gas, we will make frequent use of this fact (Young \& Freedman, 2010, p. 636)

Thus the preceding argument is meant to provide understanding to the student, and should be considered an explanation. Understanding is commonly used in University Physics to point to other passages that for the purposes of this study can be treated as explanations. It is not always clear, however, how the promised understanding will be provided. For instance the following is found in the introduction to chapter 17: 
The concepts in this chapter will help you understand the basic physics of keeping warm and cool. (Young \& Freedman, 2010, p. 551)

In this instance further indicators are needed about where in the chapter this understanding is provided and how it is provided. A partial answer to this question is that the concepts help us to answers to certain whyquestions like the one concerning children in a swimming pool mentioned above (sec 6.3.1.1).

Most instances of the word "understanding" appear in the titles of the 'Test your understanding' questions that are generously distributed throughout the whole text ${ }^{12}$. These explanation seeking questions allow the student to test whether she has gained sufficient understanding of the subject matter discussed in the preceding section to proceed to the next sections. The authors' answers to these questions are given at the end of each chapter. These answers could be relevant for the current study since they explicitly serve as guides to what an appropriate answer to an explanation seeking question looks like in the current discipline at the current level.

This leads to the more general question of how one can use the exercises in textbooks as sources of explanations.

\subsubsection{Including the exercises}

Learning to construct high quality explanations requires practice. In science educations an important part of this practice comes through solving textbook exercises and evaluating the answers. Explanations are among the kinds of answers the textbook question writer is hoping to elicit.

I will refer to an exercise that is formulated as an explicit request for an explanation or as an explanation seeking how- or why-question, as an 'Explanation Requesting Exercise' or just an ERE. Could one extend the material searched to include the exercises in order to identify the EREs and perhaps use the solution manuals containing elaborated solutions to exercises that are available for many textbooks as a source of explanations?

I believe that this approach could be useful, but it is important to be sensitive to its limitations. Introductory textbooks usually contain an abundance of exercises. University Physics for instance contains well over a hundred exercises after each chapter, and though the majority of the exercises are not EREs the sheer number of exercises means that it will be possible to get some data. The more advanced textbooks generally contain much fewer exercises than introductory textbooks and the density of EREs is also much lower.

\subsubsection{University physics}

The EREs in University Physics fall into two general groups. The first group a) is formulated as a description of a phenomenon or a result of a calculation combined with a request for an explanation. For instance (Young \& Freedman, 2010, p. 622):

\footnotetext{
${ }^{12}$ This type of questions is common in more recent introductory textbooks.
} 


\section{Explain why in a gas of $\mathbf{N}$ molecules, the number of molecules having speeds in the finite} interval $v+\Delta v$ is $\Delta N=N \int_{v}^{v+\Delta v} f(v) d v$.

Answers to this type of exercise can be taken as explanations without question.

The second group b) contains EREs where students are asked to explain their reasoning behind a certain answer. This kind of ERE is completely absent from the more advanced textbook passages that I have looked at (see next section). In the type b) exercises the main function of the word explain is to force the students to make an elaborate answer. Take for instance the following discussion question (Young \& Freedman, 2010, p. 579):

Q18.23: If the root-mean-square speed of the atoms in an ideal gas is to be doubled, by what factor must the Kelvin temperature of the gas be increased? Explain

The question posed can be answered by stating a single number ("4"). Thus if "explain" was omitted it could easily be thought that a satisfactory answer to this question is simply " 4 ". However the addition of the word explain indicates that the student has to come up with a more elaborate answer such as " 4 , because the rootmean-square speed of the atoms in an ideal gas is proportional to the square root of the Kelvin temperature of the gas".

"Why/Why not" or simply "Why?" is also often added after questions that can be answered very briefly. Thus the addition of "Why?" or "Explain" after other kinds of questions than why-questions can be seen as a clarification from the authors that the question just posed is indeed an explanation seeking question, not just what might be called a fact seeking question.

Type b) EREs are particularly interesting for two reasons. First they pose explanation seeking questions that are not why- questions. As mentioned there is some consensus that answers to this type of question should be treated as explanations, but little attention has been given to them so far. Secondly one will need knowledge about this kind of explanation seeking questions if data is to be gathered from the exercises in the more advanced textbooks ${ }^{13}$, since EREs are so rare in these, as I will illustrate below.

\subsubsection{More advanced textbooks}

Chemical Thermodynamics contains no exercises at all. I therefore made a brief search in two physics textbooks, Introduction to Electrodynamics (Griffiths, 1999) and Statistical Physics (Mandl, 1988), both aimed at slightly more advanced physics students than University Physics. Searching the exercises of two random chapters in each book ${ }^{14}$ gave a total of 65 exercises none of which contained versions of the word

\footnotetext{
${ }^{13}$ Assuming that there is data to be found. It may be that the reason why explanation seeking questions are so hard to find in more advanced textbooks is because they are not posed, but given the commonness of explanation seeking questions in everyday discourse and the consensus among philosophers and scientists that explanations are important in science I find that highly unlikely.

${ }^{14}$ Chapter 3-4 in (Mandl, 1988) and chapter 8-9 in (Griffiths, 1999)
} 
'explanation' or 'to explain'. The search for explanation seeking how- and why-questions yielded only three results, all from Introduction to Electrodynamics. Most exercises in these two books are formulated not as a question, but as a request to "find", "show" or "calculate" something. This indicates that even if an elaborated solution manual can be found to these more advanced textbooks, it may still be difficult to use the keywords discussed here, to find explanations in them.

A further complication related to the more advanced textbooks is that it is usually only the introductory textbooks that have solution manuals that elaborate on how the exercises should be solved. Most solution guides just give the result, and even the most elaborate solution manuals do not always contain solutions to the EREs, especially if the solution requires the construction of a qualitative argument. Thus the inclusion of the exercises from the more advanced textbooks might not be of much use in practice if due to time limitations it is not possible to make large scale studies of how practitioners would solve these EREs, or unless the investigator herself is capable of producing the solutions, which would require something close to contributory expertise (as opposed to interactional expertise (Collins, 2004)) for the most advanced textbooks.

All in all I find that the exercises from the introductory textbooks and their solutions could be a valuable source of explanations from the different disciplines. However it is not yet clear whether the exercises from more advanced textbooks can become as valuable, since the tools discussed in this chapter are of limited use when trying to analyze these exercises. However the results gained through the analysis of the introductory textbook exercises could provide the necessary tools for studying the more advanced exercises.

\section{Conclusions}

Philosophers' studies of scientific explanations have served as background and inspiration for studies in science education on many occasions. Although it is widely recognized that explanatory practices differ between scientific disciplines philosophers have almost exclusively focused on what similarities there may be. The aim of this chapter was to develop an empirical method for exposing possible differences in exemplar explanations given by practicing scientists to students. Empirical studies of explanations are rare in both philosophy of science and science education. The ones that exist share the assumption that the nature of explanations is best studied through the identification of a set of concrete explanations that can be used as the basis of an inductive argument. I have followed this assumption in this chapter, and thus I have not considered other possible approaches to an empirical study of explanations ${ }^{15}$.

When presenting an empirical study of explanations based on a set of concrete explanations it is important that the data collection procedure is made transparent. Since it is not essential to identify every explanation in

\footnotetext{
${ }^{15}$ An alternative approach would be to interview practicing scientists and ask them what characterizes good explanations for students or what they think are the main differences between the explanations from their discipline and explanations from other disciplines. How useful such an approach would be is an empirical question, and I am not aware that it has ever been attempted.
} 
a textbook in order to have a useful data set, and since there is no consensus on a definition of an explanation, I argued that one useful approach is to use a set of reliable linguistic indicators as the basis for gathering explanations from science textbooks. The list consisted of a number of keywords that should be fairly uncontroversial to use as identifiers of explanations. I then showed that the use of these keywords could yield a dataset that was as varied as the dataset gathered by Dagher, and the pilot study showed that the approach yields substantial amounts of data, especially from the search for explanation seeking how-questions. The pilot study also indicated that why-questions and references to understanding are more common in introductory textbooks than the advanced textbooks which produced a little less data. This highlighted that it may be important either to include larger samples of advanced textbooks in the sources or to reevaluate the search criteria after the first search, and go through the sources more than once using increasingly sophisticated criteria.

The method developed here was designed to fit a very specific purpose, and parts of the argumentation rests heavily on this specific purpose, especially the arguments for limiting the study to just textbooks. However, the usefulness of an empirical approach based on relevant sources of explanations is not dependent on the specific purposes considered here. As mentioned one could gain a very detailed picture of explanations in any scientific discipline if a combination of textbook studies and interviews was conducted. Such studies could provide an important supplement to the many case studies of explanations from different disciplines that are in the current philosophical literature.

\section{Acknowledgements}

This chapter benefitted from critical feedback from Hanne Andersen, Douglas Allchin and an anonymous reviewer. Furthermore it benefitted from insights and comments provided by the entire philosophy of contemporary science in practice group at Aarhus University. The group is supported by the Danish Council for Independent Research | Humanities.

\section{References}

Braaten, M. \& Windschitl, M. (2011): Working toward a stronger conceptualization of scientific explanation for science education. Science Education, 95: 639-669.

Chambliss, M. (2001): Analyzing science textbook materials to determine how "persuasive" they are. Theory into Practice, 40: 255-264.

Collins, H. (2004): Interactional expertise as a third kind of knowledge. Phenomenology and the Cognitive Sciences, 3: 125-143. 
Dagher, Z. (1991): Methodological decisions in interpretive research: The case of teacher explanations. In Interpretive research in science education ed., J. Gallagher, 61-83. National Association for Research in Science Teaching.

Dagher, Z., \& Cossman, G. (1992): Verbal explanations given by science teachers: Their nature and implications. Journal of Research in Science Teaching, 29: 361-374.

De Regt, H. W., S. Leonelli and \& K. Eigner, K. (2009): Scientific understanding: Philosophical perspectives. Pittsburgh, Pa.: University of Pittsburgh Press.

Draper, S. (1988): What's going on in everyday explanations. In Analysing everyday explanation: A casebook of methods, ed. C. Antaki, 15-32. London: Sage Publications.

Edgington, J. R. \& Barufaldi, J.P. (1995): How research physicists and high-school physics teachers deal with the scientific explanation of a physical phenomenon. Paper Presented at the Annual Meeting of the National Association for Research in Science Teaching, (San Francisco, CA, April 22-25, 1995).

Faye, J. (1999): Explanation explained. Synthese, 120: 61-75.

Friedman, M. (1974): Explanation and scientific understanding. The Journal of Philosophy, 71: 5-19.

Goddiksen, M. (2013): Interdisciplinarity and explanation: How interdisciplinary education calls for a new approach to research on scientific explanations. Proceedings of the $12^{\text {th }}$ biennial IHPST conference. http://archive.ihpst.net/2013-pittsburgh/conference-proceedings/

Godfrey-Smith, P. (2003): Theory and reality: An introduction to the philosophy of science. Chicago: University of Chicago Press.

Goodwin, W. (2003): Explanation in organic chemistry. Annals of the New York Academy of Sciences, 988: 141153.

Griffiths, D. J. (1999): Introduction to electrodynamics, 3. ed. Upper Saddle River, NJ: Prentice Hall.

Hempel, C. G. (1965): Aspects of scientific explanation. New York: The Free Press.

Kirkwood, J. G. \& Oppenheim, I. (1961): Chemical thermodynamics. New York: McGraw-Hill.

Kuhn, T.S. (1996): The structure of scientific revolutions, 3rd ed. Chicago: University of Chicago Press.

Lipton, P. (2009): Understanding without explanation. In Scientific understanding, ed. H. W. de Regt, S. Leonelli and K. Eigner, 43-64. Pittsburgh, Pa.: University of Pittsburgh Press. 
Machamer, P., Darden, L. \& Craver, C. (2000): Thinking about Mechanisms. Philosophy of Science, 67: 1-25.

Mahan, B. M. and Myers, R. J. (1987): University chemistry (4th ed.). Menlo Park, Calif.: Benjamin/Cummings cop.

Mandl, F. (1988): Statistical physics (2nd ed.). Chichester: John Wiley.

McMullin, E. (1993): Rationality and paradigm change in science. In World changes: Thomas Kuhn and the nature of science, ed. P. Horwich. Cambridge, Mass.: MIT Press.

Nersessian, N. J. (1995). Opening the black box: Cognitive science and history of science. Osiris, 10: 194-211.

Ogborn, J., G. Kress, I. Martins and K. McGillicuddy. (1996): Explaining Science in the Classroom. London: Open University Press.

Overton, J.A. (2013): "Explain" in scientific discourse. Synthese, 190: 1383-1405.

Peker, D., and C. Wallace. (2011): Characterizing high school students' written explanations in biology laboratories. Research in Science Education, 41: 169-191.

Rowan, K. E. (1988): A contemporary theory of explanatory writing. Written Communication, 5: 23-56.

Salmon, W. C. (1998): Why ask "why?"? An inquiry concerning scientific explanation. In Causality and Explanation, ed. W. Salmon, 125-142. New York: Oxford University Press.

Solomon, J. (1995): Higher level understanding of the nature of science. School Science Review, 76: 15-22.

Treagust, D. and A. Harrison. (1999): The genesis of Effective Scientific Explanations for the Classroom. Researching Teaching: Methodologies and Practices for Understanding Pedagogy, ed. J.L. Loughran, 28-43. London: Routledge.

Unsworth, L. (2001): Evaluating the language of different types of explanations in junior high school science texts. International Journal of Science Education, 23: 585-609.

Van Fraassen, B. C. (1980). The scientific image. Oxford: Clarendon.

Weatherall, J. O. (2011). On (some) explanations in physics. Philosophy of Science, 78: 421-447.

Woodward, J. (2011): Scientific explanation. In Stanford encyclopedia of philosophy, ed. E. N. Zalta. <http://plato.stanford.edu/archives/win2011/entries/scientific-explanation/>. Accessed May 4, 2014. 
Woody, A. I. (2003): On explanatory practice and disciplinary identity. Annals of the New York Academy of Sciences, 988: 22-29.

Woody, A. I. (2004a): Telltale signs: What common explanatory strategies in chemistry reveal about explanation itself. Foundations of Chemistry, 6: 13-43.

Woody, A. I. (2004b): More telltale signs: What attention to representation reveals about scientific explanation. Philosophy of Science, 71: 780-793.

Young, H. D. and R. D. Freedman. (2010). Sears and Zamansky's University Physics: with Modern Physics (13th int. ed.) San Francisco: Addison-Wesley. 